\title{
Quilty Effect: Its Definition and Significance, in Post-Cardiac Transplant Endomyocardial Biopsies with Focus on Our Experience
}

\author{
Kambiz Mozaffari, ${ }^{1}$ Hooman Bakhshandeh,,${ }^{1,}$ Akbar Nikpajouh, ${ }^{1}$ Nasim Naderi, ${ }^{1}$ and Ali Sadeghpour \\ Tabaei $^{1}$ \\ ${ }^{1}$ Rajaie Cardiovascular Medical and Research Center, Iran University of Medical Sciences, Tehran, IR Iran \\ "Corresponding author: Hooman Bakhshandeh, Cardiovascular Intervention Research Center, Rajaie Cardiovascular Medical and Research Center, Sciences, Vali-e-Asr St., \\ Niayesh Blvd, Tehran, IR Iran. Tel/Fax: +98-2123923138, E-mail: hooman.bakhshande@gmail.com
}

Received 2016 November 06; Revised 2016 November 29; Accepted 2016 December 21.

\begin{abstract}
Background: The recent trend of cardiac transplantation has been dramatic in our center, thus entailing the interpretation of endomyocardial biopsies in such patients.

Objectives: With this fact borne in mind, we decided to review the cases from the point of view of a certain diagnostic challenge, namely quilty lesion or effect.

Methods: From April 2010 to December 2012, 42 patients with heart transplant have undergone endomyocardial biopsies and 63 samples were acquired.

Results: Mean age of the patients was $30 \pm 15.7$ years (male/female: $34 / 8$ ). Quilty effect was seen in 8 out of 63 samples (12.7\%).

Conclusions: The incidence of quilty lesion was within the range defined for this finding. When the lesion extends to the underlying myocardial tissue, it can be a diagnostic challenge. In the eyes of an inexperienced observer, ISHLT grade 2 rejection may be diagnosed instead of invasive quilty lesion. Therefore, experienced pathologists play an important role in this regard in all the cardiovascular transplantation sites.
\end{abstract}

Keywords: Heart Transplantation, Quilty Effect, Graft Rejection, Biopsy

\section{Background}

The recent progress of cardiac transplantation has been dramatic in our center. We expected that diagnostic challenges were to be noted in the interpretation of endomyocardial biopsies of the patients who are being assessed for their rejection status histopathologically. With this fact borne in mind, we decided to review the cases from this point of view (1).

Quilty lesion or effect is one of the biggest of such challenges. It represents an endocardial inflammatory infiltrate which is seen in endomyocardial biopsies of some heart transplant patients. This term was named after the first transplant patient in whom the finding was established. It is attributed to the usage of cyclosporine in immunosuppressive regimens of transplant patients (2).

\section{Objectives}

We would like to focus on one of the most important findings in post-transplant biopsies, namely the quilty effect (1-4).

\section{Methods}

From April 2010 to December 2012, 42 heart transplant patients who referred to Rajaie Cardiovascular Medical and Research Center (RCMRC), a tertiary care center for cardiovascular patient in Tehran, Iran for endomyocardial biopsies were se;ected for this study. Sixty-three biopsy samples were acquired for monitoring acute transplant rejection in the abovementioned group. All of the patients were planned for endomyocardial biopsy based on the guidelines of "international society for heart and lung transplantation (ISHLT)" (1).

Considering the fact that majority of the patients who referred to RCMRC for biopsy were from other cardiovascular centers, we could not achieve all of their information in light of their first biopsy procedure or the interval between the procedures. Be that as it may, based on the general transplant protocol, patients are needed to undergo biopsy every month in the first year after transplantation; unless the existence of clinically suspected rejection leads to additional sampling (1).

In defined intervals, patients were examined and underwent biopsy. The myocardial tissue was obtained from the right ventricular side of interventricular sep- 
tum. There were no complications and all the patients were discharged with stable cardiovascular condition. All the biopsy samples were taken to the histopathology lab and usual processes of imbedding and staining were performed. In the end, the pathologist assessed the prepared slides and reported the final diagnosis. Here, we are not dealing with specimen inadequacy, nor are we discussing the presence of scar or adipose tissues or blood clots instead of myocardium. We did not consider viral myocarditis here either. This infection may mimic rejection pattern pathologically. There are other findings in the biopsy which may be important clinically, such as early ischemic injury and post-transplant lympho-proliferative disorders (PTLD). We considered these issues, too (1).

Data was analyzed using Mann Whitney U and Fisher's exact tests. SPSS 15 for Windows (SPSS Inc., Chicago, Illinois) was applied for the statistical analysis. P value $<0.05$ was considered as statistically significant.

\section{Results}

Forty two post-transplant patients (mean age: $30 \pm 15.7$ years (rang: 16 - 58 years), male/female: 34/8) were studied and 63 samples were obtained. Quilty effect was seen in 8 out of 63 samples (12.7\%). Patients' mean age in Quilty effect samples was $33 \pm 16.6$ years and male/female ratio was $6 / 2$. In other samples, patients' mean age was $30 \pm 15.1$ and male/female ratio was 45/10. No differences about age and sex between two groups were observed $(\mathrm{P}>0.05)$.

No other important lesions were observed (1).

\section{Discussion}

Morphologically, the lesion consists of focal flat to nodular densely cellular endocardial nodule. The infiltrate may either localize the endocardium (type A) or extend somewhat deeply into its underlying myocardial tissue (type B).

The latter can be associated with myocyte damage. Important as it is to the pathologist to differentiate type A from type B lesion, the clinical significance is not worrisome for the clinicians. Quilty lesion is mainly comprised by $\mathrm{T}$ cells, while other cell types such as B cells are seen with few macrophages and plasma cells. Small blood vessels can also be found in these infiltrates. These changes are seen in $10 \%$ to $20 \%$ of post-transplant biopsies (2).

Similarly, in our specimens, as presented in the results section, quilty effect was found in $12.7 \%$ of samples, which is within the range defined in other studies $(1,2,5)$.

The etiology of quilty lesions is unknown, but it is attributed to cyclosporine therapy. Also, its relationship to acute rejection is controversial. Lesions which are indicative of rejection may coexist in the same biopsy sample. In case quilty lesion is confined to endocardium the diagnostic challenge is not significant, but when the lesion extends to the underlying myocardial tissue, the connection between myocardial and endocardial lesions may not be evident in tangential cuts, making differentiation from acute cellular rejection difficult. In such cases it is necessary to obtain additional deeper sections to establish the diagnosis of quilty effect. Therefore, in the eyes of an inexperienced observer, ISHLT grade 2 rejection may be diagnosed instead of type B quilty lesion (2, 6-9).

As said before, it is not a clinically important issue, whether quilty lesion is restricted to the endocardium or it invades the myocardium and shows a more severe presentation. Nevertheless, it is an important issue to the pathologists and creates a challenge in the interpretation of samples. If a pathologist views only part of the biopsy with areas of myocardial invasion and damage, it is very likely to misinterpret this area as a grade $1 \mathrm{R}$ or $2 \mathrm{R}$ rejection (5-9).

The safest policy for dealing with this problem is to acquire at least three different sections from different levels of tissue. When invasive quilty lesion exists, serial sections can reveal the relation of myocardial injury to the overlying endocardium. Indeed, in this situation if we have only a tangential cut through a biopsy sample, we cannot see the relations between the myocardial infiltration and the endocardial nodules and it is very probable to report the findings as rejection.

However, the association between quilty effect and acute rejection is kind of controversial. Recent studies have shown that incidence of acute transplant rejection in patients with quilty lesions may be greater than other patients. Therefore, we usually ask the clinicians for close follow-up of transplant patients. This lesion is benign and should be differentiated from acute rejection. Further immunosuppression treatment is not needed (1).

Other findings, such as a dense infiltrate, presence of B cells and plasma cells and presence of prominent fibrovascular background can be in favor of quilty effect.

In this regard, immuno-histochemical staining demonstrates a combination of $\mathrm{B}$ and $\mathrm{T}$ cells and may confirm the diagnosis (1-9).

Other authors believe that quilty lesion may be a sign of an impending rejection or a manifestation thereof. Another question of concern was whether or not quilty lesion was capable of transforming into a post-transplant lymphoproliferative disorder (PTLD) or lymphoma. This was later proved to be irrelevant because quilty lesions consist predominantly of T cells, whereas PTLD is of B-cell origin (2).

Another bigger study reviewed the morphology of 527 
endomyocardial specimens from 46 transplant patients. This was to evaluate the significance and relation of quilty lesion with acute rejection. They concluded that the finding of isolated quilty effect may signal the prompt development of an acute rejection episode and that quilty effect is a manifestation of acute rejection, modified by many factors, such as cyclosporine treatment (10).

\subsection{Conclusion}

This study aimed to look at a certain aspect of cardiac transplantation from the point of view of a pathologist regardless of clinical findings only to highlight the importance of "quilty effect"; a term which may not be well known to many cardiologists. Quilty effect is not only mistaken for transplant rejection in myocardial samples, but it may also signify the emergence of possible severe rejections. Therefore when the term quilty effect is reported to the clinicians, the decision to reevaluate the patients and observe them closely is inferred from such a report.

\section{References}

1. Mozaffari K, Bakhshandeh H, Amin A, Naderi N, Taghavi S, OjaghiHaghighi Z, et al. Diagnostic Pitfalls and Challenges in Interpretation of Heart Transplantation Rejection in Endomyocardial Biopsies With Focus on our Experience. Res Cardiovasc Med. 2014;3(1):13986. doi: 10.5812/cardiovascmed.13986. [PubMed: 25478529].
2. Silver MD, Gotlieb AI, Schoen FJ. Cardiovascular pathology. 3rd ed. New York: Churchill Livingstone Company; 2001. pp. 727-56.

3. Costanzo MR, Dipchand A, Starling R, Anderson A, Chan M, Desai $S$, et al. The International Society of Heart and Lung Transplantation Guidelines for the care of heart transplant recipients. J Heart Lung Transplant. 2010;29(8):914-56. doi: 10.1016/j.healun.2010.05.034. [PubMed: 20643330].

4. Cunningham KS, Veinot JP, Butany J. An approach to endomyocardial biopsy interpretation. J Clin Pathol. 2006;59(2):121-9. doi: 10.1136/jcp.2005.026443. [PubMed: 16443725].

5. Stearns D. In: Sternberg's diagnostic surgical pathology. 5th ed. Sheldon LK, editor. Philadelphia, PA: Lippincott Williams and Wilkins company; 2010. pp. 1199-205.

6. Kottke-Marchant K, Ratliff NB. Endomyocardial lymphocytic infiltrates in cardiac transplant recipients. Incidence and characterization. Arch Pathol Lab Med. 1989;113(6):690-8. [PubMed: 2658911].

7. Radio SJ, McManus BM, Winters GL, Kendall TJ, Wilson JE, CostanzoNordin MR, et al. Preferential endocardial residence of B-cells in the "Quilty effect" of human heart allografts: immunohistochemical distinction from rejection. Mod Pathol. 1991;4(5):654-60. [PubMed: 1836878].

8. Joshi A, Masek MA, Brown BJ, Weiss LM, Billingham ME. "Quilty" revisited: a 10-year perspective. Hum Pathol. 1995;26(5):547-57. doi: 10.1016/0046-8177(95)90252-X. [PubMed: 7750939].

9. Fishbein MC, Bell G, Lones MA, Czer LS, Miller JM, Harasty D, et al. Grade 2 cellular heart rejection: does it exist?.J Heart Lung Transplant. 1994;13(6):1051-7. [PubMed: 7865512].

10. Pardo-Mindan FJ, Lozano MD. "Quilty effect" in heart transplantation: is it related to acute rejection?.J Heart Lung Transplant. 1991;10(6):93741. [PubMed: 1756159 ] 